\section{Rheumatoide Arthritis: Sitzzeit und Symptomatik}

O'Leary H, Larkin L, Murphy GM et al. Relationship Between Pain and Sedentary Behavior in Rheumatoid Arthritis Patients: A Cross-Sectional Study. Arthritis Care \& Research 2021; 73 : 990-997

Verbringt man seinen Tag überwiegend im Sitzen, wirkt sich das negativ auf die Gesundheit aus. Bei Patienten mit rheumatoider Arthritis (RA) sind die Auswirkungen u.a. wegen des dadurch erhöhten kardiovaskulären Risikos möglicherweise noch stärker als in der Allgemeinbevölkerung. Trotzdem führen gerade diese $\mathrm{Pa}$ tienten den größten Teil des Tages sitzende Tätigkeiten aus.

In Irland wurde nun der Zusammenhang zwischen der der Zeit, die im Sitzen verbracht wird („Sitzzeit“) und der Symptomatik der RA untersucht. Für ihre Studie konnten O'Leary und ihre Kollegen 72 Patienten (61,5 $\pm 10,5$ Jahre) gewinnen, die seit durchschnittlich 17,8 Jahren unter RA litten; ihr Krankheitsaktivitätsscore lag im Schnitt bei 11,2. Mit dem Neigungsmesser „activPAL4“ wurde über sieben Tage die Aktivität der Probanden gemessen; das Messgerät sollte jeden Tag 24 Stunden getragen werden. Die Patienten gingen anfangs davon aus ca. fünf Stunden pro Tag im Sitzen zu verbringen, tatsächlich waren es im Schnitt 8,9 Stunden (533,17 $\pm 100,1$ Minuten), d. h. 59,9\% des „wachen Tages“.

Schmerzintensität, die subjektiv angegebene Anzahl schmerzhafter Gelenke und das Vorhandensein von Fuß- und/oder Knöchelschmerzen korrelierten mit der Sitzzeit. Eine unabhängige Assoziation zwischen diesen Faktoren und der Zeit, die im Sitzen verbracht wird konnten die Autoren allerdings nicht feststellen. Höhere Level von Depression und Angst (gemessen mit der Hospital Anxiety and Depression Scale) waren assoziiert mit einer längeren Sitzzeit, während nicht artikuläre Schmerzen hingegen gar nicht damit korrelierten. Behandlungen, die auf eine Reduktion der Krankheitsaktivität abzielen, werden also wahrscheinlich die Sitzzeit beeinflussen, aber nicht die Schmerzen außerhalb von Gelenken.
Patienten, die unter Fuß- und Knöchelschmerzen litten, verbrachten signifikant mehr Zeit im Sitzen als Patienten ohne Schmerzen in diesen Regionen. Außerdem hatten sie signifikant höhere Krankheitsaktivitätsscores und litten unter stärkeren Schmerzen. Die stärkeren funktionellen Einschränkungen und das schwerere klinische Erscheinungsbild könnten eine Erklärung für die erhöhte Sitzzeit bei Patienten mit Fuß- und Knöchelschmerzen sein. Allerdings erlaubt dieses Symptom keine unabhängige Prädiktion der Sitzzeit.

Obwohl bekannt ist, dass körperliche Aktivität die RA-bedingte Fatiguesymptomatik reduzieren kann, war dieses Symptom in der vorliegenden Untersuchung nicht mit den sitzenden Tätigkeiten assoziiert. Gleiches galt für Schlafstörungen (gemessen mit dem Pittsburgh Sleep Quality Index).

FAZIT

Aufgrund der nur schwachen Korrelationen zwischen verschiedenen Aspekten der RA und der Sitzzeit können die Autoren keine klinischen Empfehlungen aus ihren Ergebnissen ableiten. Zwar spielen Schmerzen und andere RA-bedingte Faktoren offenbar eine Rolle bei sitzenden Tätigkeiten, doch signifikant scheint ihr Einfluss nicht zu sein. Insgesamt sehen die Autoren hier noch viel Forschungsbedarf.

\section{Stephanie Gräwert, Leipzig}

